\title{
Interventions for improving recovery from work (Protocol)
}

Sinval J, van Veldhoven M, Oksanen T, Azevedo LF, Atallah ÁN, Melnik T, Marôco J

Sinval J, van Veldhoven M, Oksanen T, Azevedo LF, Atallah ÁN, Melnik T, Marôco J. Interventions for improving recovery from work (Protocol).

Cochrane Database of Systematic Reviews 2021, Issue 12. Art. No.: CD014518. DOI: 10.1002/14651858.CD014518.

\section{www.cochranelibrary.com}


TABLE OF CONTENTS

ABSTRACT 1

BACKGROUND

Figure 1.

OBJECTIVES

METHODS

ACKNOWLEDGEMENTS

REFERENCES

APPENDICES

HISTORY

CONTRIBUTIONS OF AUTHORS

DECLARATIONS OF INTEREST

SOURCES OF SUPPORT

NOTES 
[Intervention Protocol]

\section{Interventions for improving recovery from work}

Jorge Sinval1,2,3,4, Marc van Veldhoven5, Tuula Oksanen 6 , Luis Filipe Azevedo7, Álvaro N Atallah ${ }^{8}$, Tamara Melnik ${ }^{8}$ João Marôco $^{3}$

1Business Research Unit (BRU-IUL), Instituto Universitário de Lisboa (ISCTE-IUL), Lisbon, Portugal. 2Faculty of Philosophy, Sciences and Languages of Ribeirão Preto, University of São Paulo, Ribeirão Preto - SP, Brazil. 3 William James Center for Research, ISPA - Instituto Universitário, Lisbon, Portugal. ${ }^{4}$ Faculdade de Medicina, Universidade de Lisboa, Lisbon, Portugal. ${ }^{5}$ Department of Human Resource Studies, Tilburg University, Tilburg, Netherlands. ${ }^{6}$ University of Eastern Finland, Kuopio, Finland. ${ }^{7}$ Center for Health Technology and Services Research (CINTESIS) \& Department of Community Medicine, Information and Health Decision Sciences (MEDCIDS), Faculty of Medicine, Porto University, Porto, Portugal. 8Brazilian Cochrane Centre, Centro de Estudos de Saúde Baseada em Evidências e Avaliação Tecnológica em Saúde, São Paulo - SP, Brazil

Contact: Jorge Sinval, jorgesinval@gmail.com.

Editorial group: Cochrane Work Group.

Publication status and date: New, published in Issue 12, 2021.

Citation: Sinval J, van Veldhoven M, Oksanen T, Azevedo LF, Atallah ÁN, Melnik T, Marôco J. Interventions for improving recovery from work (Protocol). Cochrane Database of Systematic Reviews 2021, Issue 12. Art. No.: CD014518. DOI: 10.1002/14651858.CD014518.

Copyright () 2021 The Cochrane Collaboration. Published by John Wiley \& Sons, Ltd.

\section{A B S T R A C T}

\section{Objectives}

This is a protocol for a Cochrane Review (intervention). The objectives are as follows:

To compare the effectiveness of different individual interventions in recovery from work. 


\section{B A C K G R O U N D}

\section{Description of the condition}

Recovery from work is a fundamental factor for workers' wellbeing. Recovery can be conceptualized as a process or as an outcome, and these can be considered two different aspects of the same phenomenon (Sonnentag 2009). The first facet of recovery refers to experiences and activities that create change in the strain levels, whereas the worker's physiological and psychological state after the time interval where recovery occurred is a conception of recovery as an outcome (Sonnentag 2017). Recovery from work is the process of replenishing resources after work (Hobfoll 1989). The term recovery can be defined as resuming to a prior state in which one has sufficient physical and mental resources to allow the recommencement of work tasks (Verbeek 2019). It is a process of psychophysiological unwinding and restoration after the expending of individual efforts (Craig 1992; Demerouti 2007; Geurts 2006). It occurs to recuperate from feelings of fatigue, requesting rest to allow resources to be replenished and to recover from effort investments. Through this process, workers may have their energy levels restored and are prepared to resume physical and psychological workloads. When recovery is successful, it can prevent the accumulation of fatigue that leads to serious health problems, and without it, one cannot counterbalance cognitive and physical resources gain (Gorgievski 2008). The need for recovery can be described as the perception of urgency when depletion of energy reserves happens, which leads people to feel the need to have a pause from their demands (Demerouti 2007). The recovery process occurs every time after work and can be a protector of possible negative effects of workload on workers. The lack of recovery occurs due to extended working days (Avgoustaki 2019), which do not necessarily lead to an increase in productivity. This can be seen as a symptom of an early phase of long-term strain that includes psychological distress (Mickel 2012), and that can lead to health problems, such as burnout (Gorgievski 2008; Sinval 2019).

The quality of sleep is an important factor associated with the recovery process (Sonnentag 2003). Like recovery itself, it can be both seen as a recovery process and as a recovery outcome. A proper recovery during non-work time can be accompanied with better sleep quality (Sonnentag 2009; Tucker 2008). Sonnentag 2003 suggests a possible interaction effect of sleep with leisuretime activities on feelings of recovery, alerting to the importance of including measures of sleep in studies that investigate recovery from work. Good sleep can counterbalance the lack of recovery from work, while lack of sleep can decompensate the replenishing effect of recovery after work. In addition, impaired sleep can also be considered an indicator of long-term insufficient recovery from work, which can lead to burnout (Åkerstedt 2009). Sleep is fundamental in the recovery process both in terms of quality and quantity (Fritz 2016), curtailments and disturbances in sleep can have a multiplicity of negative effects (e.g. endocrinological, immunological, metabolism), and can be linked to disease (Åkerstedt 2003).

Since overtime work is associated with the reduced opportunity for physical, mental and emotional recovery (Pencavel 2016), it is expected that occupations that require extended work days are especially demanding, for example, in small businesses where extended opening hours are necessary to keep the business running. Short-term efforts (e.g. exercise/sports and social activities) and long-term efforts (e.g. vacations) can address fatigue
(Bloom 2009), exhaustion and psychological problems such as mood issues (Mickel 2012), but seem to be short-lived (Zijlstra 2006).

Some psychometric instruments associated with the recovery from work process exist, namely, the 'Need for Recovery Scale' (NfR; van Veldhoven 2003) which measures the perceptions of need for recovery after work (van Veldhoven 2008), and was framed from the effort-recovery model. Another instrument is the 'Recovery Experience Questionnaire' (REQ; Sonnentag 2007) drawing on mood regulation and job-stress recovery literature, it assesses how individuals recuperate and unwind from work. Both the NfR and REQ scales presented good psychometric properties (Sonnentag 2007; van Veldhoven 2008). Although it is important to have measurement instruments with good validity evidence (American Educational Research Association 2014), it is not enough and one should be able to decide which intervention to choose based on evidence. So far there are no systematic reviews of interventions for improving recovery from work (to our knowledge) and reviews of that nature would be of special interest to underpin initiatives towards recovery improvement in the workplace. In this Cochrane Review, we will focus on interventions targeted at improving workrelated recovery, which should be distinguished from recovery from diseases (i.e. recovery to a state of normal functioning from a state of disease).

\section{Description of the intervention}

As suggested by Verbeek 2019, interventions for improving recovery from work can be classified into two types, individual person-directed (i.e. aiming at behavioural change) and directed at changes in the workplace (i.e. aiming at changes in work organization or in work tasks). This review will categorize interventions to further organize, classify and explore their effectiveness. The first type includes an approach to introduce behavioural changes in workers' habits, while the second is concerned with the way the workplace setting is configured towards recovery.

The person-directed interventions can be further grouped as relaxation, promotion of physical activity, recovery training and stress management. Relaxation interventions can be implemented through various techniques (i.e. yoga, listening to music, being in nature, mindfulness, progressive muscle relaxation enhanced with biofeedback or based on intervention mapping with stakeholders). An example of a relaxation intervention is a regular session by an instructor at the workplace (Coffeng 2014). An example of a promotion of physical activity intervention is a one-hour low-intensity running session performed three times a week for six consecutive weeks (de Vries 2015; de Vries 2017). An example of a recovery training intervention is an online training for better sleep in workers (six one-week sessions) that uses cognitive behavioural therapy for insomnia (e.g. stimulus control, sleep hygiene, cognitive interventions and sleep restriction) supplemented with metacognitive therapy, behavioural activation and gratitude research (Thiart 2013; Thiart 2015). An example of a stress management intervention is a web-based intervention with two components (i.e. one of problem-solving and one of emotion regulation) divided into seven sessions with modules for psychoeducation (one session), problem-solving (two sessions), emotion regulation (three sessions) and plan for the future (one session); each session could be completed in 45 to 60 minutes and consist of general information, interactive exercises, examples related to 
work, audio and video files, quizzes and downloadable mp3 files and worksheets (Ebert 2016a; Heber 2013).

Interventions related to workplace changes can be classified as participatory changes, breaks and workload changes. An example of a participatory change intervention is two 12-month phases: a phase of needs assessment and an implementation phase (Schelvis 2013). In the first phase, workers develop actions to work happily and healthily under the supervision of the heuristic method facilitator using three different steps: 10 one-hour interviews, open-ended questionnaires and group sessions (Schelvis 2017). After the last step, the facilitator informs the management team which interventions can be implemented in the next phase (Schelvis 2013). In the second phase, the changes are implemented by the management teams (Schelvis 2017). An example of a break intervention is a redesign of work-rest schedules (Beynon 2000), whereas an example of a workload changes intervention is a group of four different working schemes between two different jobs (i.e. truck driving and refuse to collect) (Kuijer 2004). The participants work one week as truck drivers only, one week as refuse collectors only, one week rotating between refuse collecting and truck driving (between days) and one week rotating between refuse collecting and truck driving (between the day) (Kuijer 2005).

\section{How the intervention might work}

There are several theoretical models that deal with recovery from work and how that process occurs (i.e. resources repletion and depletion) (Demerouti 2009; Verbeek 2019). One of them is the effort-recovery model (Meijman 1998), which states that physiological activation or fatigue are reactions to the efforts invested while working. Recovery occurs, under normal conditions, after the end of the expenditure of the efforts, and the worker is no longer exposed to work demands (Sonnentag 2007). As a consequence, the fatigue and other effects of stressful situations are diminished (Sonnentag 2001). This theoretical framework is based on exercise physiology but can be extended to tasks that demand psychological workload (Sonnentag 2001). This theory states that insufficient recovery can be a serious health risk (Geurts 2014), albeit such risks will not necessarily cause chronic workrelated problems, as long the process of recovery occurs (van Hooff 2015). The NfR psychometric instrument has its theoretical framework based on the effort-recovery theory (van Veldhoven 2003).

The conservation of resources theory suggests that workers have resources that they struggle to obtain, maintain, foster and protect (Hobfoll 1989; Hobfoll 1998; Hobfoll 2002). It is the basis for other theories of organizational stress, such as the job demandsresources model (Hobfoll 2018). The conservation of resources theory states that resource (i.e. things that individuals centrally value) loss, or the threat of such loss, leads to stress (Hobfoll 2001a; Hobfoll 2001b). It is based on four principles (Hobfoll 2018): 1. "primacy of loss principle" - resources loss is more salient than resources gain (Westman 2004); 2. "resource investment principle" - those resources must be invested in order to gain more resources, protect and recover from potential resources losses (Halbesleben 2014); 3. "gain paradox principle" - the gain of resources receives more importance in a scenario of resource loss; and 4. "desperation principle" - when resources levels are at the limit, individuals will adopt a defensive approach. Those defensive efforts will be invested in order to protect the remaining resources (Halbesleben 2014). According to this theory, if recovery is insufficient, people lose their energy/vitality, which is a valued resource in work and in life (i.e. quality of life). Such loss, or the threat of such loss, can be considered a stressor for workers. Gorgievski 2008 states that the process of resources gain and loss should be seen as a film, not as a snapshot. Such a principle can be applied to work engagement, where gaining cycles of resources can lead to more positive experiences, more risky resources investments and more capabilities to lead in work demands. Resources and work engagement can be reciprocally related (Salanova 2010). Those principles lead to three corollaries: 1 . those who have fewer resources are more exposed to vulnerabilities than those with more resources, 2. "resource loss cycles" - those of have fewer resources are more vulnerable and have more tendency to lose resources in the future and 3. "resource gain spirals" - the opposite of the second corollary, that states that those with more resources are less vulnerable and will be capable of gaining more resources in the future. However, the gain spirals tend to be weaker and to develop slowly (Hobfoll 2018). The conservation of resources theory and the effort-recovery model explain complementary processes by which recovery takes place (Sonnentag 2007). The second states that it is essential to recover from work, and avoid tasks that use the same internal resources or functional systems that are used at work, while the first states that acquiring new resources will help to restore the threatened resources (Siltaloppi 2012; Sonnentag 2001).

The allostatic load model (McEwen 1993) states that the constant forcing of physiological activation beyond its maximum capacity can lead to a cumulative effect, may conduct to illness (McEwen 2015). This happens due to the repeated attempts of the body to adjust to the stress challenges. This process is named allostasis, and it consists in the physiological change that the body's multisystems simultaneously undergo to deal with situations of stress (McEwen 1998a). Allostatic overload is seen as the wear and tear of the bodily experiences (McEwen 1993; McEwen 2003), which happen as a consequence of the repeated use of adaptive responses to stress, and of the incapability to turn on or shut off such reactions (Lupien 2015). If there is an absence of recovery, problems may emerge and the adaptive systems can start to fail, conducting to physical and mental deterioration (van Hooff 2011). Contrarily, being able to have a recovery process can be an indicator of good health (Demerouti 2009). Insufficient or incomplete recovery has been linked to allostatic load (von Thiele 2006). The allostatic load model together with the effort-recovery model provide a basis to the essential importance of this psychophysiological unwinding process (i.e. recovery) (Thiart 2013). Since the effortrecovery model does not provide a detailed description of which psychophysiological systems are essential in the recovery process, the allostatic load theory can overcome this limitation of the effortrecovery model (Geurts 2006).

Affect-regulation theory also helps to explain the understanding of recovery from work, since it refers to the mechanism of maintaining or modifying emotions or moods whose operation is dependent on the screening of affective information (Parkinson 1996). Several strategies can be employed to avoid or diminish negative mood, either cognitive or behavioural (Parkinson 1999; Webb 2012). Based on this theory, together with the conservation of resources theory, four core recovery experiences were suggested (Sonnentag 2007): psychological detachment from work, control during offjob time, relaxation and mastery experiences. The psychological detachment from work regards the mental disconnection from work (Sonnentag 2011a). Relaxation concerns a physical and 
psychological state of low activity and increased positive affect (Stone 1995). Control during off-job time refers to the level to which individuals have the discretion to choose what to do during their recovery opportunities (i.e. self-determination during leisure time and self-management) (Hahn 2011; Sonnentag 2007). Finally, mastery experiences refer to experiences that the individual has in the off-job time, and that provide challenges and opportunities to learn (Sonnentag 2011a). The four core recovery experiences are measured by four latent variables through the REQ psychometric instrument (Sanz-Vergel 2010; Sonnentag 2007). Based on a metaanalytic study (Newman 2014), two more recovery experiences were proposed, for example, meaning and affiliation. Meaning refers to individuals' needs to feel useful and have a purpose in their lives (Virtanen 2020). Affiliation concerns the need to feel closely and emotionally linked to others (Kujanpää 2021). Additionally, the authors suggested that the recovery experience control during off-job time to be replaced by autonomy, which has a broader scope, since it highlights the importance of one having perceptions of volition in general, and not only regarding the choice of one's leisure schedule (Virtanen 2020). And that the term recovery refers to recovery experience relaxation (Sonnentag 2007), which, altogether create the DRAMMA model: detachment, recovery, autonomy, mastery, meaning and affiliation (Newman 2014). Having the appropriate leisure time, and the subjective experiences during it are fundamental for the recovery from work process (Sonnentag 2017).

The cognitive activation theory of stress (CATS) states that stress response depends on acquired expectancies of the outcomes of stimuli and available responses (Ursin 2004). Thus, continuous experiences with a particular stimulus make it possible for individuals to regulate and adjust themselves (Meurs 2011). CATS distinguishes four aspects of stress (Eriksen 2017). The stress stimuli or stressor, which consists in the demands or threats to which one is exposed to. The stress experience, which involves one's appraisal of the stressor or situation and the perception of how one will deal with the stressor will lead to experiencing the stressor as pleasant or threatening, it also depends on the fourth aspect, feedback from the stress response. The non-specific, general stress response, which comprises the generic activation (alarm) stimulating wakefulness and arousal in the brain to get ready to solve the stressor or the problem. Finally, the experience of the stress response, it is the feedback obtained with the activation (arousal wakefulness) due to the stressor, and it is fundamental in the way one perceives the stress experience. The short-term activation or phasic arousal created by handling a difficult situation is not a concern for someone who is healthy (Ursin 1978), constant experiences causing arousal and alarm might be a reason for some worry, as the allostatic load theory mentions (McEwen 1998b). However, an important concern arises when there is no solution for the problem or stressor, which with humans is particularly problematic, because such unresolved problems/stressors are accompanied by sustained activation (Eriksen 2005). This concerns the incapacity to downgrade the motivational system and return to 'normal' levels, which might cause serious harm (Eriksen 2005), similar to the failed shutdown of the system suggested by the allostatic load theory (McEwen 1998b). Albeit the CATS differs from the allostatic load model in the extent that it takes into account the importance of the cognitive variable (Eriksen 2005) in the presence of rumination, for example, persistent negative expectancies and thoughts (Brosschot 2004). This theory helps us to understand the harm caused by lack of recovery from work in the way that the continuous failure to develop recovery from work and the cognitions associated with persistent rumination can lead to serious consequences in health.

Drawing on this latter theory, finally there is the stressordetachment model (Sonnentag 2011b), which also draws on the previously mentioned allostatic load model. The stressordetachment model assumes the recovery processes occurs mainly via the detachment from work when being at home. The detachment from work can replenish resources and diminish strain symptoms (Sonnentag 2016). This model states that the stressorstrain process is mediated and moderated by psychological detachment. Lack of the detachment from work is considered to be the core mechanism that can explain the fatigue and other symptoms of an unsuccessful recovery process (Sonnentag 2011b). The mediation process occurs when the job stressors obstruct the detachment from work process, influencing strain and workers' well-being (Sonnentag 2015). The moderation process suggests that psychological detachment affects the impacts of job stressors on poor well-being and strain, lower levels of psychological detachment will lead to increased impact of job stressors on strain and well-being (Sonnentag 2018). The initial model was later extended, adding that the job stressors' impacts on psychological detachment are moderated by personal and job resources (Schulz 2019), higher levels of both can buffer the influence of job stressors on psychological detachment (Sonnentag 2015).

Numerous interventions aim to enhance recovery from work, being individual person-directed or being targeted towards changes at the workplace level (Verbeek 2019). As depicted in Figure 1, there are several options of individual person-directed type (Verbeek 2019). Relaxation techniques are based on the principle that those interventions can increase one's feeling of recovery (Siltaloppi 2012). Physical activity is founded on the principle that such effort will increase stress resilience and improve psychological detachment (de Vries 2017). Recovery training interventions stem from the recovery experiences (Sonnentag 2007) where increasing work engagement or happiness will in turn increase recovery (Feicht 2013; Mastenbroek 2015). Stress management interventions are based on problem-based coping and emotion regulation, which are expected to improve recovery (Ebert 2016b; Richardson 2008). Some interventions about the work organization level have been compiled (Verbeek 2019). Participatory changes interventions are based on the principle that more employee resources (e.g. more support for personal and family lives, more control over their work time) can result in increased recovery and civility (Hammer 2016). Interventions of introducing breaks stem from resourcebased principles, where more break time can lead to higher reductions of fatigue, better recovery and improved performance (Lim 2016; Mathiassen 1996). Interventions regarding workload changes act through alterations as the task rotation can lead to improved recovery and better musculoskeletal outcomes (Kuijer 2005). For the purpose of the systematic review, we will consider only individual person-directed interventions. These types of intervention are easier to implement, since they are aimed at individual behavioural change, instead of depending on the organization to put it into practice (i.e. changes in the work organization or work tasks). 
Figure 1. Logic model of the intervention

\section{Interventions for improving recovery from work}

\section{Individual person-directed interventions (ie, aiming at behavoral change)}

- Relaxation

-Promotion of physical activity

-Recovery training

-Stres management

\section{Outcome measurements}

\section{*Fatigue \\ -Perfomance \\ -Physiological}

\section{Why it is important to do this review}

Improved workplace health is a prerequisite to attain the United Nations Sustainable Development Goals, particularly, Goals 1, 3 and 8 (United Nations 2017).The World Health Organization's global plan of action on workers' health emphasized the importance of generating evidence about the health of the working population and using such evidence to produce policies and concrete actions that assure the improvement of workers' health (WHO
2013). Research in workers' health and safety should be a priority (WHO 2016), since the investment in this specific field guarantees a diminution of the direct and indirect costs in terms of health, improves workers' productivity, and raises companies' competitiveness (International Labour Office 2011). In order to accomplish what is expected from them, workers spend energy and effort to achieve work goals (Bennett 2018). Work conditions and demands might deplete workers' cognitive, physical and emotional resources (Meijman 1998), which need to be replaced 
after work. The restoration of depleted resources after working hours is essential to protect workers from the possible negative results of workplace stress and to maintain good health (Ebert 2015). Recovery from work is important in the sense that it allows the psychophysiological individual systems to return and stabilize at a baseline level, where no special demands are made on the individual (Geurts 2006). The information gathered through this review can be used to diminish the associated risks with poor recovery from work. This can be particularly important in small businesses where the workload can be difficult to cope with, mainly, when efforts are needed in order to allow company survival.

There are some previous reviews related to recovery from work; one scope review (Verbeek 2019), one literature review (Fehrmann 2014), and three meta-analytic studies (Bennett 2018; Steed 2021; Wendsche 2017). None of these directly addressed the issue of the specific types of interventions employed in improve recovery from work through a systematic revision. Additionally, there are two meta-analyses: one focusing on a specific recovery experience (i.e. detachment from work; Karabinski 2021), and one approaching individual-focused occupational health interventions (Estevez Cores 2021). The scope review revealed that there is uncertainty about the existing evidence, reporting studies that presented opposing results (Verbeek 2019). Since that evidence has not been combined in a systematic review, there is justification to do so (Melnik 2011). Additionally, a meta-analysis - if there are enough suitable studies - will provide evidence about the effects of specific interventions (Verbeek 2012).

\section{O B J E C T I VES}

To compare the effectiveness of different individual interventions in recovery from work.

\section{METHODS}

\section{Criteria for considering studies for this review}

\section{Types of studies}

We will include eligible randomized controlled trials (RCTs) regardless of whether participants are assigned to groups individually or in clusters (cluster-RCTs). We will include cross-over RCTs, but only data from the first active treatment phase.

\section{Types of participants}

We will include studies conducted with currently employed healthy adults of working age (from 18 to 65 years old) and who were exposed to physical or cognitive (or both) workload. We will exclude people with diseases or morbidities that can confound the efficacy of the interventions on the improvement of recovery from work. Specific attention will be given to particular target groups of the interventions as the individuals working in small businesses/selfemployed can have a workload difficult to regulate.

\section{Types of interventions}

We will include studies that evaluate person-directed interventions for improving workers' process of recovery from work. We will consider different interventions (as described in How the intervention might work) following Verbeek 2019 classification.
- Individual person-directed.

- Relaxation.

- Physical activity.

- Recovery training.

- Stress management.

We will include trials that compare the effectiveness of the active intervention with (preferably) active intervention not aimed at increasing recovery (i.e. for an attention effect) or with no intervention or with another active intervention from those that we listed. We will exclude studies that focus only on stress management to avoid overlap with an existing systematic review on the topic (Ruotsalainen 2015). We will exclude studies that focus on occupation-specific recovery standards.

\section{Types of outcome measures}

We will produce effects sizes for the different type of interventions, and forest plots and heterogeneity tests to quantify the effectiveness of the different type of interventions.

\section{Primary outcomes}

- Recovery from work (e.g. need for recovery, NfR psychometric instrument (van Veldhoven 2003); recovery experiences, REQ psychometric instrument (Sonnentag 2007)).

- Performance (e.g. auditory oddball task (Lim 2016)).

- Fatigue (i.e. Borg scale (Borg 1982)).

We chose these primary outcomes because they were suggested by Verbeek 2019 in their scope review (Levac 2010), where they broadly divided the outcomes into three categories (i.e. potential consequences of the lack of recovery, personal or job resources, and workload and other draining of resources).

\section{Secondary outcomes}

- Employee satisfaction with the intervention.

- Cost-effectiveness of the intervention.

- Physiological. We will accept any measure based on physiological parameters (i.e. cardiovascular and musculoskeletal).

- Adverse events associated with the intervention.

We will accept any measurement of recovery based on psychometric instruments that showed adequate validity evidence. Potential meta-analysis mediators and moderators might be tested (e.g. recovery experiences, temporal scope of the interventions: at work, after work, unspecific depending on the question to be made) (Shadish 1991). We will consider the following follow-up times for outcome measurement: short-term defined as less than three months after the intervention has been completed, mediumterm defined as between three months and less than 12 months, and long-term defined as 12 months or longer.

Reporting one or more of the secondary outcomes listed here in the trials is not an inclusion criterion for the review. Adverse events are not included, as Verbeek 2019 found no reported adverse events. 


\section{Search methods for identification of studies}

\section{Electronic searches}

We will conduct a systematic literature search to identify all published and unpublished trials that can be considered eligible for inclusion in this review. We will adapt the search strategy we developed for MEDLINE for use in the other electronic databases (see Appendix 1). We will impose no restriction on language of publication. We will arrange for the translation of key sections of potentially eligible non-English language papers or we will arrange people who are proficient in the publications' languages to fully assess them for potential inclusion in the review, as necessary.

We will search the following electronic databases from inception for identifying potential studies:

- Cochrane Central Register of Controlled Trials (CENTRAL) (the Cochrane Library);

- MEDLINE (PubMed) (Appendix 1);

- Embase (embase.com);

- PsycINFO (ProQuest);

- OSH-UPDATE (www.oshupdate.com);

- CINAHL (EBSCO).

We will also conduct a search for unpublished trials in ClinicalTrials.gov (www.ClinicalTrials.gov) and the World Health Organization trials portal (www.who.int/ictrp/en/).

\section{Searching other resources}

We will check the reference lists of all included trials and relevant systematic reviews to identify additional trials missed from the original electronic searches (e.g. unpublished or in-press citations). We will contact experts in the field to identify additional unpublished materials.

\section{Data collection and analysis}

\section{Selection of studies}

We will conduct the selection of eligible studies in two stages using the review management program Covidence (Covidence). First, two review authors (JS and JM) will independently screen titles and abstracts of all the potentially relevant studies we find with our systematic search to exclude studies that clearly do not fulfil the criteria for inclusion. The same review authors will code them as 'include' (eligible or potentially eligible) or 'exclude'. At this stage, we will exclude all references that clearly do not fulfil our inclusion criteria or that do fulfil our exclusion criteria.

At the second stage, we will retrieve the full-text study reports or publications for all references that we included at the first stage. Tworeview authors (JS and, MVV) will assess the full-text and identify studies for inclusion,so that all full-text reports are assessed independently by two review authors. We will resolve any disagreements through discussion or, if required, we will consult a third review author (LA). At this stage, we will include all references that do fulfil our inclusion criteria.

We will record reasons for exclusion of the ineligible studies assessed as full texts in a 'Characteristics of excluded studies' table. We will identify and exclude duplicates (at the first stage), and collate multiple reports of the same study so that each study, rather than each report, is the unit of interest in the review. We will record the selection process in sufficient detail to complete a PRISMA study flow diagram.

Should our systematic searches identify studies conducted by authors of this review, we will avoid conflicts of interest by ensuring all decisions concerning inclusion and exclusion made by review authors who were not involved with the study.

\section{Data extraction and management}

Two review authors (JS and JM) will extract the following data on study characteristics and outcomes using Covidence.

- Methods: study design, total duration of study, study location, study setting, withdrawals and date of study.

- Participants: number, mean age or age range, sex/gender, severity of condition, diagnostic criteria if applicable, inclusion criteria and exclusion criteria.

- Interventions: description of intervention, comparison, duration, intensity, content of both intervention and control condition, and cointerventions.

- Outcomes: primary and secondary outcomes specified and collected, and time points reported.

- Notes: funding for trial and notable conflicts of interest of trial authors.

We will check all included trials for a process evaluation, and describe the core elements of the process (e.g. attendance, compliance, dose delivered, dose received, etc.).

Two review authors (JS and JM) will independently extract outcome data from included studies. We will note in the 'Characteristics of included studies' table if outcome data were not reported in a usable way. We will resolve disagreements by consensus or by involving a third review author (LA). One review author (JS) will transfer data into the Review Manager Web (Review Manager Web 2019). We will double-check that data are entered correctly by comparing the data presented in the systematic review with the study reports. A second review author (JM) will spot-check data for accuracy against the trial report. Should we decide to include studies published in one or more languages in which our author team is not proficient, we will arrange for a native speaker or someone sufficiently qualified in each foreign language to complete a data extraction form for us.

\section{Assessment of risk of bias in included studies}

Two review authors (JS and JM) will independently assess risk of bias for each outcome separately using the criteria outlined in the Cochrane Handbook for Systematic Reviews of Interventions (Higgins 2019a). We will resolve any disagreements by discussion or by involving another review author (TM). We will assess the risk of bias according to the following domains.

- Random sequence generation.

- Allocation concealment.

- Blinding of participants and personnel.

- Blinding of outcome assessment.

- Incomplete outcome data.

- Selective outcome reporting.

- Other bias. 
For cluster-RCTs, we will assess (in addition to the basic RCT risk of bias domains listed above):

- Recruitment bias;

- Baseline differences between clusters.

For cross-over RCTs, we will assess (in addition to the basic RCT risk of bias domains listed above):

- Bias resulting from carry-over effects.

We will grade each potential risk of bias as high, low or unclear, and provide a quote from the study report together with a justification for our judgement in the risk of bias table. We will add the additional domains for other study designs to the risk of bias table. We will summarize the risk of bias judgements across different studies for each of the domains listed. We will consider blinding separately for different key outcomes where necessary. Where information on risk of bias relates to unpublished data or correspondence with a trial author, we will note this in the risk of bias table.

Should our systematic searches identify studies conducted by authors of this review, we will avoid conflicts of interest by ensuring assessment of risk of bias is made by review authors who were not involved with the study.

\section{Overall risk of bias at study level}

We will judge a study to have a high risk of bias overall when we judge one or more domains to have a high risk of bias. Conversely, we will judge a study to have a low risk of bias when we judge low risk of bias for all domains.

When considering treatment effects, we will take into account the risk of bias for the studies that contribute to that outcome.

\section{Assessment of bias in conducting the systematic review}

We will conduct the review according to this published protocol and report any deviations from it in the 'Differences between protocol and review' section of the systematic review.

\section{Measures of treatment effect}

We will enter the outcome data for each study into the data tables in RevMan Web to calculate the treatment effects (Review Manager Web 2019). We will use risk ratios for dichotomous outcomes, and mean differences (when studies use the same scale) or standardized mean differences (when studies use difference scales) for continuous outcomes, or other types of data (e.g. correlational measures) as reported by the authors of the studies.

If only effect estimates and their 95\% confidence intervals or standard errors are reported in studies, we will enter these data into RevMan Web using the generic inverse variance method (Review Manager Web 2019). We will ensure that higher scores for continuous outcomes have the same meaning for the particular outcome, explain the direction to the reader and report where the directions were reversed if this was necessary. When the results cannot be entered in either way, we will describe them in the 'Characteristics of included studies' table, or in additional tables or narratively.

\section{Unit of analysis issues}

\section{Cluster-randomized trials}

We will include cluster-RCTs if proper adjustment for the intracluster correlation can be conducted in accordance with the Cochrane Handbook for Systematic Reviews of Interventions (Higgins 2019a). If studies report sufficient data to be included in the meta-analysis but do not make an allowance for the design effect, we will calculate the design effect based on a fairly large assumed intracluster correlation of 0.10 . We base this assumption of 0.10 being a realistic estimate by analogy on studies about implementation research (Campbell 2001). We will follow the methods stated in the Cochrane Handbook for Systematic Reviews of Interventions for the calculations (Higgins 2019a).

\section{Cross-over trials}

We will include trials employing a cross-over design in the review, but we will only use data from the first active treatment phase. When cross-over trials report continuous outcomes with which the authors have not reported a paired analysis, we will perform a paired analysis based on a reported or imputed correlation between the outcomes of the intervention and the control condition, as advised in the Cochrane Handbook for Systematic Reviews of Interventions (Higgins 2019a). For dichotomous outcomes, we will adjust the confidence intervals for the paired analysis according to Elbourne 2002.

\section{Trials with multiple treatment groups}

Multiple-arm trials (those with more than two intervention arms) can pose analytical problems in pairwise meta-analyses. For trials with more than two eligible arms, we will manage data in this review as follows.

\section{Multiple experimental intervention groups versus a single control group}

If studies compare multiple eligible experimental interventions with a single control group, we will split the control group to enable pairwise comparisons.

\section{Dealing with missing data}

We will contact investigators or study sponsors to verify key study characteristics and obtain missing numerical outcome data where possible (e.g. when a study is identified as abstract only).

We also plan to conduct best-/worse-case scenarios for the clinical response outcome, in which we will assume that dropouts in the active treatment group had positive outcomes and those in the control group had negative outcomes (best-case scenario), and that dropouts in the active treatment group had negative outcomes and those in the control group had positive outcomes (worst-case scenario), thus providing boundaries for the observed treatment effect. If a large amount of information is missing, we will give these best-/worst-case scenarios greater emphasis in the presentation of results.

We will analyze missing continuous data on an endpoint basis, including only participants with a final assessment, or by using the last observation carried forward (LOCF) to the final assessment, if trial authors report LOCF data. If numerical outcome data are missing, such as standard deviations or correlation coefficients, and they cannot be obtained from the authors, we will calculate 
them from other available statistics such as $\mathrm{P}$ values according to the methods described in the Cochrane Handbook for Systematic Reviews of Interventions (Higgins 2019a).

\section{Assessment of heterogeneity}

We will assess the clinical homogeneity of the results of included studies based on similarity of population, intervention, outcome and follow-up.

- We will consider populations as similar when they are similar regarding their type and baseline level of workload: cognitive, physical, or both.

- We will consider interventions as similar when they fit inside the same type of intervention (as stated in Types of interventions, where four different intervention types were presented).

- For each of the referred psychometric instruments (e.g. NfR, REQ) original versions, we will consider their correspondent adaptations as similar measures (i.e. different countries, cultures, etc.).

- We will regard follow-up times of short, medium and long term as different (as defined in Secondary outcomes).

We will use the $I^{2}$ statistic to measure heterogeneity among the trials in each analysis. If we identify substantial heterogeneity, we will report it and explore possible causes by prespecified subgroup analysis. We will consider heterogeneity as substantial if $\mathrm{I}^{2}$ is above $50 \%$.

\section{Assessment of reporting biases}

If we are able to pool more than five trials in any single metaanalysis, we will create and examine a funnel plot to explore possible small-study biases.

\section{Data synthesis}

We will pool data from studies we judge to be clinically homogeneous, as defined in Assessment of heterogeneity, using Review Manager Web (Review Manager Web 2019). If more than one study provides usable data in any single comparison, we will perform meta-analyses. We will use a random-effects model because we believe that the type of intervention and study designs included will always lead to heterogeneity. When $\mathrm{I}^{2}$ is higher than $75 \%$, we will not pool results of studies in meta-analyses.

Where multiple trial arms are reported in a single trial, we will include only the relevant arms. If two comparisons (e.g. intervention one versus no intervention and intervention two versus no intervention) are combined in the same meta-analysis, we will halve the control group to avoid double-counting. We will list all intervention arms, even if they are irrelevant to the review, in the 'Characteristics of included studies' table.

\section{Subgroup analysis and investigation of heterogeneity}

We plan to conduct the following two subgroup analyses:
- the use of different measures to assess recovery (i.e. recovery, need for recovery and fatigue); the existence of different baseline workloads (i.e. if higher levels of baseline workload correspond to higher effectiveness of the interventions).

We will use the $x^{2}$ test to test for subgroup interactions in Review Manager Web (Review Manager Web 2019).

\section{Sensitivity analysis}

We will perform sensitivity analyses defined a priori to assess the robustness of our conclusions. This will involve:

- studies with low risk of bias versus studies with high risk of bias.

We will also perform sensitivity analyses to check how our assumptions influence the conclusions of the review.

\section{Reaching conclusions}

We will base our conclusions only on findings from the quantitative or narrative synthesis of included studies for this review. We will avoid making recommendations for practice based on more than just the evidence, such as values and available resources. Our implications for research will suggest priorities for future research and outline what the remaining uncertainties are in the area.

\section{Summary of findings and assessment of the certainty of the evidence}

We will create summary of findings tables only for the main comparisons that are of best interest to decision makers (Schünemann 2019a). We will report all outcomes for these comparisons. We will use the five GRADE considerations (study limitations, consistency of effect, imprecision, indirectness and publication bias) to assess the certainty of a body of evidence as it relates to the studies that contribute data to the metaanalyses for the prespecified outcomes. We will use methods and recommendations described in Chapters 8 and 15 of the Cochrane Handbook for Systematic Reviews of Interventions using GRADEpro GDT software (GRADEpro GDT; Higgins 2019b; Schünemann 2019b). We will justify all decisions to downgrade or upgrade the certainty of the evidence using footnotes.

\section{ACKNOWLEDGEMENTS}

We thank Julitta Boschman, Managing Editor, Jan Hoving, Coordinating Editor, Jani Ruotsalainen, former Managing Editor, and Jos Verbeek, Advisor, and former Co-ordinating Editor, from Cochrane Work Review Group for their help in all stages of the current review. We also thank external peer referees Johannes Wendsche and Jessica de Bloom for their comments and Anne Lawson for copy editing the text. The methods section is based on the standard Cochrane Work Group protocol template. We thank the peer referees for their useful comments to help improve this protocol. 


\section{RE F E R E N C E S}

\section{Additional references}

\section{Åkerstedt 2003}

Åkerstedt T, Nilsson PM. Sleep as restitution: an introduction. Journal of Internal Medicine 2003;254(1):6-12. [DOI: 10.1046/ j.1365-2796.2003.01195.x]

\section{Åkerstedt 2009}

Åkerstedt T, Nilsson PM, Kecklund G. Sleep and recovery. In: Sonnentag S, Perrewé PL, Ganster DC, editors(s). Current Perspectives on Job-Stress Recovery. Bingley (UK): Emerald, 2009:205-47. [DOI: 10.1108/S1479-3555(2009)0000007009]

\section{American Educational Research Association 2014}

American Educational Research Association, American Psychological Association, National Council on Measurement in Education. Standards for Educational and Psychological Testing. Washington (DC): American Psychological Association, 2014.

\section{Avgoustaki 2019}

Avgoustaki A, Frankort HT. Implications of work effort and discretion for employee well-being and careerrelated outcomes: an integrative assessment. ILR Review 2019;72(3):636-61. [DOI: 10.1177/0019793918804540]

\section{Bennett 2018}

Bennett AA, Bakker AB, Field JG. Recovery from work-related effort: a meta-analysis. Journal of Organizational Behavior 2018;39(3):262-75. [DOI: 10.1002/job.2217]

\section{Beynon 2000}

Beynon C, Burke J, Doran D, Nevill A. Effects of activityrest schedules on physiological strain and spinal load in hospital-based porters. Ergonomics 2000;43(10):1763-70. [DOI: 10.1080/001401300750004168]

\section{Bloom 2009}

Bloom J, Kompier M, Geurts S, Weerth C, Taris TW, Sonnentag S. Do we recover from vacation? Meta-analysis of vacation effects on health and well-being. Journal of Occupational Health 2009;51(1):13-25. [DOI: 10.1539/joh.K8004]

\section{Borg 1982}

Borg GA. Psychophysical bases of perceived exertion. Medicine \& Science in Sports \& Exercise 1982;14(5):377-81. [DOI: 10.1249/00005768-198205000-00012]

\section{Brosschot 2004}

Brosschot JF, Thayer JF. Worry, perseverative thinking and health. In: Nyklícek I, Temoshok L, Vingerhoets A, editors(s). Emotional Expression and Health: Advances in Theory, Assessment and Clinical Applications. London (UK): Routledge, 2004:99-115. [DOI: 10.4324/9780203484104-16]

\section{Campbell 2001}

Campbell MK, Mollison J, Grimshaw JM. Cluster trials in implementation research: estimation of intracluster correlation coefficients and sample size. Statistics in Medicine 2001;20(3):391-9. [DOI: 10.1002/1097-0258(20010215)20:3\%3C391::AIDSIM800\%3E3.0.CO;2-Z]

\section{Coffeng 2014}

Coffeng JK, Boot CR, Duijts SF, Twisk JW, van Mechelen W, Hendriksen IJ. Effectiveness of a worksite social \& physical environment intervention on need for recovery, physical activity and relaxation; results of a randomized controlled trial. PloS One 2014;9(12):1-26. [DOI: 10.1371/journal.pone.0114860]

\section{Covidence [Computer program]}

Veritas Health Innovation Covidence. Melbourne, Australia: Veritas Health Innovation, 2021. Available at www.covidence.org.

\section{Craig 1992}

Craig A, Cooper RE. Symptoms of acute and chronic fatigue. In: Smith AP, Jones DM, editors(s). Handbook of Human Performance: State and Trait. Vol. 3. London (UK): Academic Press, 1992:289-339. [DOI: 10.1016/ B978-0-12-650353-1.50017-4]

\section{Demerouti 2007}

Demerouti E, Taris TW, Bakker AB. Need for recovery, homework interference and performance: is lack of concentration the link? Journal of Vocational Behavior 2007;71(2):204-20. [DOI: 10.1016/j.jvb.2007.06.002]

\section{Demerouti 2009}

Demerouti E, Bakker AB, Geurts SA, Taris TW. Daily recovery from work-related effort during non-work time. In: Sonnentag $\mathrm{S}$, Perrewé PL, Ganster DC, editors(s). Current Perspectives on Job-Stress Recovery. Bingley (UK): Emerald, 2009:85-123. [DOI: 10.1108/S1479-3555(2009)0000007006]

\section{de Vries 2015}

de Vries JD, Van Hooff ML, Geurts SA, Kompier MA. Efficacy of an exercise intervention for employees with work-related fatigue: study protocol of a two-arm randomized controlled trial. BMC Public Health 2015;15(1):1-13. [DOI: 10.1186/s12889-015-2434-6]

\section{de Vries 2017}

de Vries JD, van Hooff ML, Guerts SA, Kompier MA. Exercise to reduce work-related fatigue among employees: a randomized controlled trial. Scandinavian Journal of Work, Environment \& Health 2017;43(4):337-49. [DOI: 10.5271/sjweh.3634]

\section{Ebert 2015}

Ebert DD, Berking M, Thiart H, Riper H, Laferton JA, Cuijpers P, et al. Restoring depleted resources: efficacy and mechanisms of change of an Internet-based unguided recovery training for better sleep and psychological detachment from work. Health Psychology 2015;34(Suppl):1240-51. [DOI: 10.1037/hea0000277]

\section{Ebert 2016a}

Ebert DD, Lehr D, Heber E, Riper H, Cuijpers P, Berking M. Internet- and mobile-based stress management for employees with adherence-focused guidance: efficacy and mechanism of 
change. Scandinavian Journal of Work, Environment \& Health 2016;42(5):382-94. [DOI: 10.5271/sjweh.3573]

\section{Ebert 2016b}

Ebert DD, Heber E, Berking M, Riper H, Cuijpers P, Funk B, et al. Self-guided Internet-based and mobile-based stress management for employees: results of a randomised controlled trial. Occupational and Environmental Medicine 2016;73(5):315-23. [DOI: 10.1136/oemed-2015-103269]

\section{Elbourne 2002}

Elbourne DR, Altman DG, Higgins JP, Curtin F, Worthington HV, Vail A. Meta-analysis involving cross-over trials: methodological issues. International Journal of Epidemiology 2002;31(1):140-9. [DOI: 10.1093/ije/31.1.140]

\section{Eriksen 2005}

Eriksen HR, Murison R, Pensgaard AM, Ursin H. Cognitive Activation Theory of Stress (CATS): from fish brains to the Olympics. Psychoneuroendocrinology 2005;30(10):933-8. [DOI: 10.1016/j.psyneuen.2005.04.013]

\section{Eriksen 2017}

Eriksen HR. The Cognitive Activation Theory of Stress (CATS) in occupational health. In: Rossi AM, Meurs JA, Perrewé PL, editors(s). Stress and Quality of Working Life: Conceptualizing and Assessing Stress. Charlotte (NC): Information Age Publishing, 2017:41-63.

\section{Estevez Cores 2021}

Estevez Cores S, Sayed AA, Tracy DK, Kempton MJ. Individualfocused occupational health interventions: a meta-analysis of randomized controlled trials. Journal of Occupational Health Psychology 2021;26(3):189-203. [DOI: 10.1037/ocp0000249]

\section{Fehrmann 2014}

Fehrmann C, Depenbrock F. Recovery from work-related stress: a literature review. Maastricht Student Journal of Psychology and Neuroscience 2014;3:85-96.

\section{Feicht 2013}

Feicht T, Wittmann M, Jose G, Mock A, von Hirschhausen E, Esch T. Evaluation of a seven-week web-based happiness training to improve psychological well-being, reduce stress, and enhance mindfulness and flourishing: a randomized controlled occupational health study. Evidence-Based Complementary and Alternative Medicine 2013;2013:1-14. [DOI: $10.1155 / 2013 / 676953$ ]

\section{Fritz 2016}

Fritz C, Crain T. Recovery from work and employee sleep: understanding the role of experiences and activities outside of work. In: Barling J, Barnes CM, Carleton E, Wagner DT, editors(s). Work and Sleep: Research Insights for the Workplace. Oxford (UK): Oxford University Press, 2016:55-76. [DOI: 10.1093/ acprof:oso/9780190217662.003.0004]

\section{Geurts 2006}

Geurts SA, Sonnentag S. Recovery as an explanatory mechanism in the relation between acute stress reactions and chronic health impairment. Scandinavian Journal of
Work, Environment \& Health 2006;32(6):482-92. [DOI: 10.5271/ sjweh.1053]

\section{Geurts 2014}

Geurts SA. Recovery from work during off-job time. In: Bauer GF, Hämmig O, editors(s). Bridging Occupational, Organizational and Public Health: a Transdisciplinary Approach. Dordrecht (Netherlands): Springer, 2014:193-208. [DOI: 10.1007/978-94-007-5640-3_12]

\section{Gorgievski 2008}

Gorgievski MJ, Hobfoll SE. Work can burn us out or fire us up: conservation of resources in burnout and engagement. In: Halbesleben JR, editors(s). Handbook of Stress and Burnout in Health Care. New York (NY): Nova Science, 2008:7-22.

\section{GRADEpro GDT [Computer program]}

McMaster University (developed by Evidence Prime) GRADEpro GDT. Hamilton (ON): McMaster University (developed by Evidence Prime), 2015. Available at gradepro.org.

\section{Hahn 2011}

Hahn VC, Binnewies C, Sonnentag S, Mojza EJ. Learning how to recover from job stress: effects of a recovery training program on recovery, recovery-related self-efficacy, and well-being. Journal of Occupational Health Psychology 2011;16(2):202-16. [DOI: 10.1037/a0022169]

\section{Halbesleben 2014}

Halbesleben JR, Neveu JP, Paustian-Underdahl SC, Westman M. Getting to the "COR": understanding the role of resources in conservation of resources theory. Journal of Management 2014;40(5):1334-64. [DOI: 10.1177/0149206314527130]

\section{Hammer 2016}

Hammer LB, Johnson RC, Crain TL, Bodner T, Kossek EE, Davis KD, et al. Intervention effects on safety compliance and citizenship behaviors: evidence from the work, family, and health study. Journal of Applied Psychology 2016;101(2):190-208. [DOI: 10.1037/apl0000047]

\section{Heber 2013}

Heber E, Ebert DD, Lehr D, Nobis S, Berking M, Riper H. Efficacy and cost-effectiveness of a web-based and mobile stress-management intervention for employees: design of a randomized controlled trial. BMC Public Health 2013;13(1):1-12. [DOI: 10.1186/1471-2458-13-655]

\section{Higgins 2019a}

Higgins JP, Thomas J, Chandler J, Cumpston M, Li T, Page MJ, et al, editor(s). Cochrane Handbook for Systematic Reviews of Interventions. 2nd edition. Chichester (UK): John Wiley \& Sons, 2019.

\section{Higgins 2019b}

Higgins JP, Savović J, Page MJ, Elbers RG, Sterne JA. Assessing risk of bias in a randomized trial. In: Higgins JP, Thomas J, Chandler J, Cumpston M, Li T, Page MJ, et al, editors(s). Cochrane Handbook for Systematic Reviews of Interventions. 2 edition. Chichester (UK): John Wiley \& Sons, 2019:205-28. 


\section{Hobfoll 1989}

Hobfoll SE. Conservation of resources: a new attempt at conceptualizing stress. American Psychologist 1989;44(3):513-24. [DOI: 10.1037/0003-066X.44.3.513]

\section{Hobfoll 1998}

Hobfoll SE. Stress, Culture, and Community: the Psychology and Philosophy of Stress. Boston (MA): Springer, 1998. [DOI: 10.1007/978-1-4899-0115-6]

\section{Hobfoll 2001a}

Hobfoll SE, Shirom A. Conservation of resources theory: applications to stress and management in the workplace. In: Golembiewski RT, editors(s). Handbook of Organizational Behavior. New York (NY): Marcel Dekker, 2001:57-80.

\section{Hobfoll 2001b}

Hobfoll SE. The influence of culture, community, and the nested-self in the stress process: advancing conservation of resources theory. Applied Psychology 2001;50(3):337-421. [DOI: 10.1111/1464-0597.00062]

\section{Hobfoll 2002}

Hobfoll SE. Social and psychological resources and adaptation. Review of General Psychology 2002;6(4):307-24. [DOI: 10.1037/1089-2680.6.4.307]

\section{Hobfoll 2018}

Hobfoll SE, Halbesleben J, Neveu JP, Westman M. Conservation of resources in the organizational context: the reality of resources and their consequences. Annual Review of Organizational Psychology and Organizational Behavior 2018;5(1):103-28. [DOI: 10.1146/annurevorgpsych-032117-104640]

\section{International Labour Office 2011}

International Labour Office. In: ILO introductory report: global trends and challenges on occupational safety and health. XIX World Congress on Safety and Health at Work; 2011 Sept 11-15; Istanbul (Turkey). Geneva (Switzerland): International Labour Organization, 2011.

\section{Karabinski 2021}

Karabinski T, Haun VC, Nübold A, Wendsche J, Wegge J. Interventions for improving psychological detachment from work: a meta-analysis. Journal of Occupational Health Psychology 2021;26(3):224-42. [DOI: 10.1037/ocp0000280]

\section{Kuijer 2004}

Kuijer PP, de Vries WH, van der Beek AJ, van Dieën JH, Visser B, Frings-Dresen MH. Effect of job rotation on work demands, workload, and recovery of refuse truck drivers and collectors. Human Factors 2004;46(3):437-48. [DOI: 10.1518/ hfes.46.3.437.50403]

\section{Kuijer 2005}

Kuijer PP, van der Beek AJ, van Dieën JH, Visser B, FringsDresen $\mathrm{MH}$. Effect of job rotation on need for recovery, musculoskeletal complaints, and sick leave due to musculoskeletal complaints: a prospective study among refuse collectors. American Journal of Industrial Medicine 2005;47(5):394-402. [DOI: 10.1002/ajim.20159]

\section{Kujanpää 2021}

Kujanpää M, Syrek C, Lehr D, Kinnunen U, Reins JA, de Bloom J. Need satisfaction and optimal functioning at leisure and work: a longitudinal validation study of the DRAMMA model. Journal of Happiness Studies 2021;22(2):618-707. [DOI: 10.1007/ s10902-020-00247-3]

\section{Levac 2010}

Levac D, Colquhoun H, O'Brien KK. Scoping studies: advancing the methodology. Implementation Science 2010;5(1):1-9. [DOI: 10.1186/1748-5908-5-69]

\section{$\operatorname{Lim} 2016$}

Lim J, Kwok K. The effects of varying break length on attention and time on task. Human Factors 2016;58(3):472-81. [DOI: 10.1177/0018720815617395]

\section{Lupien 2015}

Lupien SJ, Ouellet-Morin I, Hupbach A, Tu MT, Buss C, Walker D, et al. Beyond the stress concept: allostatic load - a developmental biological and cognitive perspective. In: Cicchetti D, Cohen DJ, editors(s). Developmental Psychopathology: Developmental Neuroscience. 2 edition. Hoboken (NJ): John Wiley \& Sons, 2015:578-628. [DOI: 10.1002/9780470939390.ch14]

\section{Mastenbroek 2015}

Mastenbroek NJ, van Beukelen P, Demerouti E, Scherpbier AJ, Jaarsma AD. Effects of a 1 year development programme for recently graduated veterinary professionals on personal and job resources: a combined quantitative and qualitative approach. BMC Veterinary Research 2015;11(1):1-13. [DOI: 10.1186/s12917-015-0627-y]

\section{Mathiassen 1996}

Mathiassen SE, Winkel J. Physiological comparison of three interventions in light assembly work: reduced work pace, increased break allowance and shortened working days. International Archives of Occupational and Environmental Health 1996;68(2):94-108. [DOI: 10.1007/BF00381241]

\section{McEwen 1993}

McEwen BS, Stellar E. Stress and the individual: mechanisms leading to disease. Archives of Internal Medicine 1993;153(18):2093-101. [DOI: 10.1001/ archinte.1993.00410180039004]

\section{McEwen 1998a}

McEwen BS. Protective and damaging effects of stress mediators. New England Journal of Medicine 1998;338(3):171-9. [DOI: 10.1056/NEJM199801153380307]

\section{McEwen 1998b}

McEwen BS. Stress, adaptation, and disease: allostasis and allostatic load. Annals of the New York Academy of Sciences 1998;840(1):33-44. [DOI: 10.1111/j.1749-6632.1998.tb09546.x] 


\section{McEwen 2003}

McEwen BS, Wingfield JC. The concept of allostasis in biology and biomedicine. Hormones and Behavior 2003;43(1):2-15. [DOI: 10.1016/S0018-506X(02)00024-7]

\section{McEwen 2015}

McEwen BS, Bowles NP, Gray JD, Hill MN, Hunter RG, Karatsoreos IN, et al. Mechanisms of stress in the brain. Nature Neuroscience 2015;18(10):1353-63. [DOI: 10.1038/nn.4086]

\section{Meijman 1998}

Meijman TF, Mulder G. Psychological aspects of workload. In: de Wolff C, Drenth PJ, Henk T, editors(s). A Handbook of Work and Organizational Psychology: Work Psychology. Vol. 2. Hove (UK): Psychology Press, 1998:5-33. [DOI: 10.4324/9780203765425-6]

\section{Melnik 2011}

Melnik T, Atallah ÁN, editor(s). Evidence-Based Psychology: Scientific Evidence of the Effectiveness of Psychotherapy [Psicologia Baseada em Evidências: Provas Científicas da Efetividade da Psicoterapia]. São Paulo (Brazil): Livraria Santos, 2011.

\section{Meurs 2011}

Meurs JA, Perrewé PL. Cognitive activation theory of stress: an integrative theoretical approach to work stress. Journal of Management 2011;37(4):1043-68. [DOI: 10.1177/0149206310387303]

\section{Mickel 2012}

Mickel AE, Dallimore EJ. Work stress: help me get my feet back on the ground. In: Reilly NP, Sirgy MJ, Gorman CA, editors(s). Work and Quality of Life: Ethical Practices in Organizations. Dordrecht (Netherlands): Springer, 2012:65-78. [DOI: 10.1007/978-94-007-4059-4_4]

\section{Newman 2014}

Newman DB, Tay L, Diener E. Leisure and subjective well-being: a model of psychological mechanisms as mediating factors. Journal of Happiness Studies 2014;15(3):555-78. [DOI: 10.1007/ s10902-013-9435-x]

\section{Parkinson 1996}

Parkinson B, Totterdell P, Briner RB, Reynolds S. Changing Moods: the Psychology of Mood and Mood Regulation. London (UK): Longman, 1996.

\section{Parkinson 1999}

Parkinson B, Totterdell P. Classifying affect-regulation strategies. Cognition and Emotion 1999;13(3):277-303. [DOI: 10.1080/026999399379285]

\section{Pencavel 2016}

Pencavel J. Recovery from work and the productivity of working hours. Economica 2016;83(332):545-63. [DOI: 10.1111/ ecca.12206]

\section{Review Manager Web 2019 [Computer program]}

The Cochrane Collaboration Review Manager Web (RevMan Web). The Cochrane Collaboration, 2019. Available at revman.cochrane.org.

\section{Richardson 2008}

Richardson KM, Rothstein HR. Effects of occupational stress management intervention programs: a meta-analysis. Journal of Occupational Health Psychology 2008;13(1):69-93. [DOI: 10.1037/1076-8998.13.1.69]

\section{Ruotsalainen 2015}

Ruotsalainen JH, Verbeek JH, Mariné A, Serra C. Preventing occupational stress in healthcare workers. Cochrane Database of Systematic Reviews 2015;4:1-152. [DOI: 10.1002/14651858.CD002892.pub5]

\section{Salanova 2010}

Salanova M, Schaufeli WB, Xanthopoulou D, Arnold BB. The gain spiral of resources and work engagement: sustaining a positive worklife. In: Bakker AB, Leiter MP, editors(s). Work Engagement: a Handbook of Essential Theory and Research. Hove (UK): Routledge, 2010. [DOI: 10.4324/9780203853047-13]

\section{Sanz-Vergel 2010}

Sanz-Vergel Al, Sebastián J, Rodríguez-Muñoz A, Garrosa E, Moreno-Jiménez B, Sonnentag S. Adaptation of the "Recovery Experience Questionnaire" in a Spanish sample. Psicothema 2010;22(4):990-6. [PMID: 21044543]

\section{Schelvis 2013}

Schelvis RM, Oude HK, Wiezer NM, Blatter BM, van Genabeek JA, Bohlmeijer ET, et al. Design of the bottom-up Innovation project - a participatory, primary preventive, organizational level intervention on work-related stress and well-being for workers in Dutch vocational education. BMC Public Health 2013;13(1):1-15. [DOI: 10.1186/1471-2458-13-760]

\section{Schelvis 2017}

Schelvis RM, Wiezer NM, van der Beek AJ, Twisk JW, Bohlmeijer ET, Oude HK. The effect of an organizational level participatory intervention in secondary vocational education on work-related health outcomes: results of a controlled trial. BMC Public Health 2017;17(1):1-14. [DOI: 10.1186/ s12889-017-4057-6]

\section{Schulz 2019}

Schulz AD, Schöllgen I, Fay D. The role of resources in the stressor-detachment model. International Journal of Stress Management 2019;26(3):306-14. [DOI: 10.1037/str0000100]

\section{Schünemann 2019a}

Schünemann HJ, Higgins JP, Vist GE, Glasziou P, Akl EA, Skoetz N, et al. Completing 'summary of findings' tables and grading the certainty of the evidence. In: Higgins JP, Thomas J, Chandler J, Cumpston M, Li T, Page MJ, et al, editors(s). Cochrane Handbook for Systematic Reviews of Interventions. 2nd edition. Chichester (UK): John Wiley \& Sons, 2019:375-402.

\section{Schünemann 2019b}

Schünemann HJ, Vist GE, Higgins JP, Santesso N, Deeks JJ, Glasziou $P$, et al. Interpreting results and drawing conclusions. In: Higgins JP, Thomas J, Chandler J, Cumpston M, Li T, Page MJ, et al, editors(s). Cochrane Handbook for Systematic Reviews of Interventions. 2nd edition. Chichester (UK): John Wiley \& Sons, 2019:403-31. 


\section{Shadish 1991}

Shadish WR, Sweeney RB. Mediators and moderators in meta-analysis: there's a reason we don't let dodo birds tell us which psychotherapies should have prizes. Journal of Consulting and Clinical Psychology 1991;59(6):883-93. [DOI: 10.1037/0022-006X.59.6.883]

\section{Siltaloppi 2012}

Siltaloppi M, Kinnunen U, Feldt T, Tolvanen A. Development of need for recovery from work over one year: a person-centered approach. Anxiety, Stress and Coping 2012;25(1):23-42. [DOI: 10.1080/10615806.2010.540649]

\section{Sinval 2019}

Sinval J, Queirós C, Pasian SR, Marôco J. Transcultural adaptation of the Oldenburg Burnout Inventory (OLBI) for Brazil and Portugal. Frontiers in Psychology 2019;10:1-28. [DOI: 10.3389/fpsyg.2019.00338]

\section{Sonnentag 2001}

Sonnentag S. Work, recovery activities, and individual wellbeing: a diary study. Journal of Occupational Health Psychology 2001;6(3):196-210. [DOI: 10.1037/1076-8998.6.3.196]

\section{Sonnentag 2003}

Sonnentag S. Recovery, work engagement, and proactive behavior: a new look at the interface between nonwork and work. Journal of Applied Psychology 2003;88(3):518-28. [DOI: 10.1037/0021-9010.88.3.518]

\section{Sonnentag 2007}

Sonnentag S, Fritz C. The Recovery Experience Questionnaire: development and validation of a measure for assessing recuperation and unwinding from work. Journal of Occupational Health Psychology 2007;12(3):204-21. [DOI: 10.1037/1076-8998.12.3.204]

\section{Sonnentag 2009}

Sonnentag S, Geurts SA. Methodological issues in recovery research. In: Sonnentag S, Perrewé PL, Ganster DC, editors(s). Current Perspectives on Job-Stress Recovery. Bingley (UK): Emerald, 2009:1-36. [DOI: 10.1108/ S1479-3555(2009)0000007004]

\section{Sonnentag 2011a}

Sonnentag S, Niessen C, Neff A. Recovery: nonwork experiences that promote positive states. In: Spreitzer GM, Cameron KS, editors(s). Oxford Handbook of Positive Organizational Scholarship. New York (NY): Oxford University Press, 2011:867-81. [DOI: 10.1093/oxfordhb/9780199734610.013.0066]

\section{Sonnentag 2011b}

Sonnentag S. Recovery from fatigue: the role of psychological detachment. In: Ackerman PL, editors(s). Cognitive Fatigue: Multidisciplinary Perspectives on Current Research and Future Applications. Washington (DC): American Psychological Association, 2011:253-72. [DOI: 10.1037/12343-012]

\section{Sonnentag 2015}

Sonnentag S, Fritz C. Recovery from job stress: the stressordetachment model as an integrative framework. Journal of
Organizational Behavior 2015;36(S1):S72-S103. [DOI: 10.1002/ job.1924]

\section{Sonnentag 2016}

Sonnentag S, Kühnel J. Coming back to work in the morning: psychological detachment and reattachment as predictors of work engagement. Journal of Occupational Health Psychology 2016;21(4):379-90. [DOI: 10.1037/ocp0000020]

\section{Sonnentag 2017}

Sonnentag S, Venz L, Casper A. Advances in recovery research: what have we learned? What should be done next? Journal of Occupational Health Psychology 2017;22(3):365-80. [DOI: 10.1037/ocp0000079]

\section{Sonnentag 2018}

Sonnentag S, Fritz C. Recovery from work. In: Ones DS, Anderson N, Viswesvaran C, Sinangil HK, editors(s). The SAGE Handbook of Industrial, Work and Organizational Psychology: Managerial Psychology and Organizational Approaches. 3rd edition. London (UK): SAGE, 2018:471-82. [DOI: 10.4135/9781473914964.n21]

\section{Steed 2021}

Steed LB, Swider BW, Keem S, Liu JT. Leaving work at work: a meta-analysis on employee recovery from work. Journal of Management 2021;47(4):867-97. [DOI: 10.1177/0149206319864153]

\section{Stone 1995}

Stone AA, Kennedy-Moore E, Neale JM. Association between daily coping and end-of-day mood. Health Psychology 1995;14(4):341-9. [DOI: 10.1037/0278-6133.14.4.341]

\section{Thiart 2013}

Thiart H, Lehr D, Ebert DD, Sieland B, Berking M, Riper H. Log in and breathe out: Efficacy and cost-effectiveness of an online sleep training for teachers affected by work-related strain - study protocol for a randomized controlled trial. Trials 2013;14(1):1-10. [DOI: 10.1186/1745-6215-14-169]

\section{Thiart 2015}

Thiart H, Lehr D, Ebert DD, Berking M, Riper H. Log in and breathe out: Internet-based recovery training for sleepless employees with work-related strain - results of a randomized controlled trial. Scandinavian Journal of Work, Environment \& Health 2015;41(2):164-74. [DOI: 10.5271/sjweh.3478]

\section{Tucker 2008}

Tucker P, Dahlgren A, Akerstedt T, Waterhouse J. The impact of free-time activities on sleep, recovery and well-being. Applied Ergonomics 2008;39(5):653-62. [DOI: 10.1016/ j.apergo.2007.12.002]

\section{United Nations 2017}

United Nations. Resolution adopted by the General Assembly on 6 July 2017, A/RES/71/313. ggim.un.org/documents/ a_res_71_313.pdf (accessed prior to 16 September 2021). 


\section{Ursin 1978}

Ursin H, Baade E, Levine S, editor(s). Psychobiology of Stress: a Study of Coping Men. New York (NY): Academic Press, 1978. [DOI: 10.1016/B978-0-127-09250-8.X5001-4]

\section{Ursin 2004}

Ursin H, Eriksen HR. The cognitive activation theory of stress. Psychoneuroendocrinology 2004;29(5):567-92. [DOI: 10.1016/ S0306-4530(03)00091-X]

\section{van Hooff 2011}

van Hooff ML, Geurts SA, Beckers DG, Kompier MA. Daily recovery from work: the role of activities, effort and pleasure. Work and Stress 2011;25(1):55-74. [DOI: $10.1080 / 02678373.2011 .570941]$

\section{van Hooff 2015}

van Hooff ML, Geurts SA. Need satisfaction and employees' recovery state at work: a daily diary study. Journal of Occupational Health Psychology 2015;20(3):377-87. [DOI: 10.1037/a0038761]

\section{van Veldhoven 2003}

van Veldhoven M, Broersen S. Measurement quality and validity of the "Need for Recovery Scale". Occupational and Environmental Medicine 2003;60:i3-9. [DOI: 10.1136/ oem.60.suppl_1.i3]

\section{van Veldhoven 2008}

van Veldhoven M. Need for recovery after work: an overview of construct, measurement and research. In: Houdmont J, Leka S, editors(s). Occupational Health Psychology: European Perspectives on Research, Education and Practice. 3rd edition. Vol. 3. Nottingham (UK): Nottingham University Press, 2008:1-25.

\section{Verbeek 2012}

Verbeek JH, Ruotsalainen JH, Hoving JL. Synthesizing study results in a systematic review. Scandinavian Journal of Work, Environment \& Health 2012;38(3):282-90. [DOI: 10.5271/ sjweh.3201]

\section{Verbeek 2019}

Verbeek J, Ruotsalainen J, Laitinen J, Korkiakangas E, Lusa S, Mänttäri S, Oksanen T. Interventions to enhance recovery in healthy workers; a scoping review. Occupational Medicine 2019;69(1):54-63. [DOI: 10.1093/occmed/kqy141]

\section{Virtanen 2020}

Virtanen A, de Bloom J, Kinnunen U. Relationships between recovery experiences and well-being among younger and older teachers. International Archives of Occupational and Environmental Health 2020;93(2):213-27. [DOI: 10.1007/ s00420-019-01475-8]

\section{von Thiele 2006}

von Thiele U, Lindfors P, Lundberg U. Self-rated recovery from work stress and allostatic load in women. Journal of Psychosomatic Research 2006;61(2):237-42. [DOI: 10.1016/ j.jpsychores.2006.01.015]

\section{Webb 2012}

Webb TL, Miles E, Sheeran P. Dealing with feeling: a metaanalysis of the effectiveness of strategies derived from the process model of emotion regulation. Psychological Bulletin 2012;138(4):775-808. [DOI: 10.1037/a0027600]

\section{Wendsche 2017}

Wendsche J, Lohmann-Haislah A. A meta-analysis on antecedents and outcomes of detachment from work. Frontiers in Psychology 2017;7:1-24. [DOI: 10.3389/fpsyg.2016.02072]

\section{Westman 2004}

Westman M, Hobfoll SE, Chen S, Davidson OB, Laski S. Organizational stress through the lens of conservation of resources (COR) theory. In: Perrewe PL, Ganster DC, editors(s). Exploring Interpersonal Dynamics. Bingley (UK): Emerald, 2004:167-220. [DOI: 10.1016/S1479-3555(04)04005-3]

\section{WHO 2013}

World Health Organization. WHO global plan of action on workers' health (2008-2017): baseline for implementation. www.who.int/occupational_health/activities/flyer_universal_ health_coverage2013.pdf (accessed prior to 16 September 2021).

\section{WHO 2016}

World Health Organization. Global Strategy on Human Resources for Health: Workforce 2030. Geneva (Switzerland): World Health Organization, 2016.

\section{Zijlstra 2006}

Zijlstra FR, Sonnentag S. After work is done: psychological perspectives on recovery from work. European Journal of Work and Organizational Psychology 2006;15(2):129-38. [DOI: 10.1080/13594320500513855]

\section{APPENDICES}

\section{Appendix 1. MEDLINE search strategy}

\#1

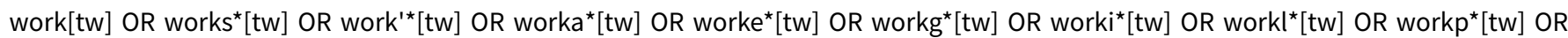
occupation $^{\star}[\mathrm{tw}]$

\#2

(Recover*[tw] OR recuperate*[tw] OR unwind*[tw]) AND (“from work" [tw] OR "after work" [tw] OR "need for" [tw] OR "need to"[tw] OR "from fatigue" [tw] OR "from job-stress"[tw] OR physiological[tw]) 
Feeling* AND ("being recovered” OR "being refreshed” OR energized)

\#4

“conservation of resources"[tw] OR “depletion of resources"[tw] OR "personal resources”[tw] OR "resource recovery"[tw] OR “replenish resources"[tw] OR "internal resources"

\#5

"stress-recovery balance"[tw] OR "effort recovery model”[tw] OR "Conservation of resources theory"[tw] OR "effort expenditure"[tw]

\#6

"Fatigue countermeasures"[tw] OR "Disengage from work"[tw] OR "Psychological detachment"[tw] OR "Mental detachment"[tw] OR "Restoration of depleted resources" [tw] OR "Recovery training"[tw] OR "Healthy restorative behaviours" [tw] OR "Recreational activities" [tw] OR "Boundary tactics" [tw] OR "Recovery modalities" [tw] OR "Recovery experience questionnaire" [tw] OR "Off-job activities" [tw] OR "Mastery and control" [tw] OR "Experience of control" [tw] OR "Mood repair" [tw] OR "Diversionary strategies" [tw] OR "Work engagement strategies" [tw] OR "Mastery oriented strategies" [tw] OR "Relaxation oriented strategies"[tw]

\#7

(randomized controlled trial[pt] OR controlled clinical trial[pt] OR randomized[tiab] OR placebo[tiab] OR drug therapy[sh] OR randomly[tiab] OR trial[tiab] OR groups[tiab] NOT (animals [mh] NOT humans [mh]))

\#8

\#1 AND (\#2 OR \#3 OR \#4 OR \#5 OR \#6) AND \#7

\section{HISTORY}

\begin{tabular}{lll}
\hline Date & Event & Description \\
\hline 8 August 2018 & Amended & Edits in the theoretical framework. \\
\hline
\end{tabular}

\section{CONTRIBUTIONSOF AUTHORS}

Conceiving the protocol: JS, MVV, TO, LFA, TM, ÁNA, JM.

Designing the protocol: JS, MVV, TM.

Co-ordinating the protocol: JS.

Designing search strategies: JS.

Writing the protocol: JS.

Providing general advice on the protocol: JS, TM, LFA, MVV.

\section{DECLARATIONS OF INTEREST}

JS: none.

MVV is one of the original authors of the NfR psychometric instrument (van Veldhoven 2003).

TO: none.

LFA: none.

ANA: none.

TM: none. 
JM: none.

\section{SOURCES OF SUPPORT}

\section{Internal sources}

- Business Research Unit (BRU-IUL), Instituto Universitário de Lisboa (ISCTE-IUL), Portugal

This work was supported by Fundação para a Ciência e a Tecnologia, grant UIDB/00315/2020.

\section{External sources}

- Fundação para a Ciência e Tecnologia, Portugal

This work was produced with the support of INCD and it was funded by FCT I.P. under the project Advanced Computing Project CPCA/ A0/7417/2020, platform Stratus.

\section{NOT ES}

Parts of the methods section and Appendix 1 of this protocol are based on a standard template established by the Cochrane Work Review Group. 